\title{
3-CONVEX FUNCTIONS AND GENERALIZATIONS OF AN INEQUALITY OF HARDY-LITTLEWOOD-PÓLYA
}

\author{
Sadia Khalid, Josip PeČarić and Marjan Praljak \\ GC University, Pakistan and University of Zagreb, Croatia
}

\begin{abstract}
In this paper, we present some generalizations of an inequality of Hardy-Littlewood-Pólya. We give the $n$-exponential convexity and log-convexity of the functions associated with the linear functionals defined as the non-negative differences of the generalized inequalities and prove the monotonicity property of the generalized Cauchy means obtained via these functionals. Finally, we give several examples of the families of functions for which the results can be applied.
\end{abstract}

\section{Introduction and Preliminaries}

The following theorem is given in the famous Hardy-Littlewood-Pólya book [3, Theorem 134].

THEOREM 1.1. If $f$ is a convex and continuous function defined on $[0, \infty)$ and $a_{k}, k \in \mathbb{N}$ are non-negative and non-increasing, then

$$
f\left(\sum_{k=1}^{n} a_{k}\right) \geq f(0)+\sum_{k=1}^{n}\left(f\left(k a_{k}\right)-f\left((k-1) a_{k}\right)\right) .
$$

If $f^{\prime}$ is a strictly increasing function, there is equality only when $a_{k}$ are equal up to a certain point and then zero. If $f$ is concave, then (1.1) holds in the reverse direction.

An example of above theorem is given below (see [3, Theorem 134]).

2010 Mathematics Subject Classification. 26A24, 26A48, 26A51, $26 \mathrm{D} 15$.

Key words and phrases. Non-increasing sequence in weighted mean, convex function, 3 -convex function, $n$-exponential and logarithmic convexity, mean value theorems, divided difference. 
THEOREM 1.2. Let $a_{k} \geq 0$ and assume that the sequence $\left(a_{k}, k \in \mathbb{N}\right)$ is non-increasing. If $s>1$, then we have

$$
\left(\sum_{k=1}^{n} a_{k}\right)^{s} \geq \sum_{k=1}^{n} a_{k}^{s}\left(k^{s}-(k-1)^{s}\right) .
$$

If $0<s<1$, then (1.2) holds in the reverse direction.

Inequality (1.1) is of great interest and has been generalized in many different ways by various mathematicians.

In 1995, inequality (1.2) was improved by J. Pečarić and L. E. Persson in [9] and this improvement is given below:

THEOREM 1.3. If the sequence $\left(a_{k}>0, k \in \mathbb{N}\right)$ is non-increasing in mean i.e if

$$
\frac{1}{n} \sum_{k=1}^{n} a_{k} \geq \frac{1}{n+1} \sum_{k=1}^{n+1} a_{k}, \quad n \in \mathbb{N},
$$

where $\left(a_{k}, k \in \mathbb{N}\right) \subset \mathbb{R}$ and if $s$ is a real number such that $s>1$, then

$$
\left(\sum_{k=1}^{\infty} a_{k}\right)^{s} \geq \sum_{k=1}^{\infty} a_{k}^{s}\left(k^{s}-(k-1)^{s}\right),
$$

holds. If $0<s<1$, then (1.3) holds in the reverse direction.

It is well known and easy to see that if a sequence $\left(a_{k}, k \in \mathbb{N}\right)$ is nonincreasing, then it is also non-increasing in mean but the reverse implications don't hold in general. This means that Theorem 1.3 is a genuine generalization of Theorem 1.2.

In 1986, G. Bennett ([2]) proved a weighted version of inequality (1.2) in the following form: if $a_{k}(k=1, \ldots, n)$ are non-negative and non-increasing and $p_{k} \geq 0$ for each $k=1, \ldots, n$ with $P_{k}=\sum_{i=1}^{k} p_{i}(k=1, \ldots, n)$, then for any real number $s>1$,

$$
\left(\sum_{k=1}^{n} p_{k} a_{k}\right)^{s} \geq\left(p_{1} a_{1}\right)^{s}+\sum_{k=2}^{n} a_{k}^{s}\left(P_{k}^{s}-P_{k-1}^{s}\right)
$$

holds. If $0<s<1$, then (1.4) holds in the reverse direction.

In 1997, C. Jardas, J. Pečarić, R. Roki and N. Sarapa presented the generalizations of these inequalities $((1.3)$ and its reverse) in [5]. As a consequence some inequalities for entropies of discrete probability distributions are also presented in [5]. Some basic properties of entropies of probability distributions can be found in [7].

The following result was proved in [5]. 
Theorem 1.4. Let $a_{k}>0(k=1, \ldots, n)$ be real numbers and $S_{k}=$ $\sum_{i=1}^{k} a_{i}(k=1, \ldots, n)$. Then for all $s, 0<s<1$ or $s>2$, we have

$$
\begin{aligned}
& \sum_{k=1}^{n} a_{k}{ }^{s}\left(k^{s}-(k-1)^{s}\right) \\
& \quad+s \sum_{k=2}^{n} a_{k}^{s-1}\left(S_{k-1}-(k-1) a_{k}\right)\left(k^{s-1}-(k-1)^{s-1}\right) \\
& \quad \leq\left(\sum_{k=1}^{n} a_{k}\right)^{s} \\
& \quad \leq \sum_{k=1}^{n} a_{k}^{s}\left(k^{s}-(k-1)^{s}\right)+s \sum_{k=2}^{n}\left(S_{k-1}-(k-1) a_{k}\right)\left(S_{k}^{s-1}-S_{k-1}^{s-1}\right) .
\end{aligned}
$$

For all $s, 1<s<2$, the opposite inequalities hold in (1.5). Equalities hold in (1.5) if and only if $a_{1}=a_{2}=\ldots=a_{n}$.

The following theorem was proved in [5] as a consequence of Theorem 1.4.

THeOREM 1.5. Let $p_{k}>0(k=1, \ldots, n)$ be a probability distribution with entropy $H=-\sum_{i=1}^{n} p_{i} \log p_{i}$ and $P_{k}=\sum_{i=1}^{k} p_{i}(k=1, \ldots, n)$. Then we have

$$
\begin{aligned}
H+ & \sum_{k=2}^{n}\left((k-1) p_{k}-P_{k-1}\right)(\log k-\log (k-1)) \\
& \leq \sum_{k=2}^{n} F(k-1) p_{k} \\
& \leq H+\sum_{k=2}^{n}\left((k-1) p_{k}-P_{k-1}\right)\left(\log P_{k}-\log P_{k-1}\right),
\end{aligned}
$$

where $F(x)=(x+1) \log (x+1)-x \log x(x>0)$. Equalities hold in (1.6) if $p_{k}=\frac{1}{n}(k=1, \ldots, n)$.

In order to obtain our general results we need some definitions.

Definition 1.6. A sequence $\left(a_{k}, k \in \mathbb{N}\right) \subset \mathbb{R}$ is non-increasing in weighted mean, if

$$
\frac{1}{P_{n}} \sum_{k=1}^{n} p_{k} a_{k} \geq \frac{1}{P_{n+1}} \sum_{k=1}^{n+1} p_{k} a_{k}, \quad n \in \mathbb{N},
$$

where $a_{k}$ and $p_{k}(k \in \mathbb{N})$ are real numbers such that $p_{k}>0(k \in \mathbb{N})$ with $P_{k}=\sum_{i=1}^{k} p_{i}(k \in \mathbb{N})$.

A sequence $\left(a_{k}, k \in \mathbb{N}\right) \subset \mathbb{R}$ is non-decreasing in weighted mean, if opposite inequality holds in (1.7). 
In a similar way we can define when a finite sequence $\left(a_{k}, k=1, \ldots, n\right) \subset$ $\mathbb{R}$ is non-increasing in weighted mean or non-decreasing in weighted mean.

REMARK 1.7. It is easy to see that a sequence $\left(a_{k}, k \in \mathbb{N}\right)$ is nonincreasing in weighted mean (non-decreasing in weighted mean) if and only if $\sum_{i=1}^{k-1} p_{i} a_{i} \geq P_{k-1} a_{k}\left(\sum_{i=1}^{k-1} p_{i} a_{i} \leq P_{k-1} a_{k}\right)$ for $k=2,3, \ldots$

The following property of convex function will be useful further (see $[8$, p.2]).

Proposition 1.8. A function $f: I \rightarrow \mathbb{R}$ is convex on $I$ if

$$
f\left(x_{1}\right)\left(x_{3}-x_{2}\right)+f\left(x_{2}\right)\left(x_{1}-x_{3}\right)+f\left(x_{3}\right)\left(x_{2}-x_{1}\right) \geq 0
$$

holds for all $x_{1}, x_{2}, x_{3} \in I$ such that $x_{1}<x_{2}<x_{3}$.

Proposition 1.9. If $f$ is a convex function on an interval $I$ and $x_{1} \leq$ $y_{1}, x_{2} \leq y_{2}, x_{1} \neq x_{2}, y_{1} \neq y_{2}$, then the following inequality is valid:

$$
\frac{f\left(x_{2}\right)-f\left(x_{1}\right)}{x_{2}-x_{1}} \leq \frac{f\left(y_{2}\right)-f\left(y_{1}\right)}{y_{2}-y_{1}} .
$$

If the function $f$ is concave, the inequality reverses (see [8, p.2]).

LEMMA 1.10. A differentiable function of one variable is convex on an interval $I$ if and only if the function lies above all of its tangents i.e

$$
f(y) \geq f(x)+(y-x) f^{\prime}(x) .
$$

By choosing the points in the reverse, we have

$$
f(x) \geq f(y)+(x-y) f^{\prime}(y) .
$$

On combining (1.9) and (1.10), we have

$$
f(y)+(x-y) f^{\prime}(y) \leq f(x) \leq f(y)+(x-y) f^{\prime}(x) .
$$

The $n$ th-order divided difference of a function $f:[a, b] \rightarrow \mathbb{R}$ at mutually distinct points $y_{0}, y_{1}, \ldots, y_{n} \in[a, b]$ is defined recursively by

$$
\begin{aligned}
& {\left[y_{i} ; f\right]=f\left(y_{i}\right), \quad i=0, \ldots, n,} \\
& {\left[y_{0}, \ldots, y_{n} ; f\right]=\frac{\left[y_{1}, \ldots, y_{n} ; f\right]-\left[y_{0}, \ldots, y_{n-1} ; f\right]}{y_{n}-y_{0}} .}
\end{aligned}
$$

The value $\left[y_{0}, \ldots, y_{n} ; f\right]$ is independent of the order of the points $y_{0}, \ldots, y_{n}$. This definition may be extended to include the case in which some or all the points coincide (see [8, p.14]).

The following definition of a real-valued convex function is characterized by $n t h$-order divided difference (see [8, p.15]).

Definition 1.11. A function $f:[a, b] \rightarrow \mathbb{R}$ is said to be $n$-convex $(n \geq 0)$ if and only if for all choices of $(n+1)$ distinct points $y_{0}, \ldots, y_{n} \in[a, b]$, we have $\left[y_{0}, \ldots, y_{n} ; f\right] \geq 0$. 
In Section 2, we present generalizations of Theorems 1.4 and 1.5. We define linear functionals as the non-negative differences of the generalized inequalities and give mean value theorems for the linear functionals. In Section 3, we give definitions and results which will be needed later. Further, we investigate the $n$-exponential convexity and log-convexity of the functions associated with the linear functionals and also deduce Lyapunovtype inequalities for these functionals. We also prove the monotonicity property of the generalized Cauchy means obtained via these functionals. Finally, in Section 4 we give several examples of the families of functions for which the obtained results can be applied.

\section{Main Results}

Our first main result is the generalization of Theorems 1.4 and 1.5.

TheOREM 2.1. Let $a_{k}>0$ and $p_{k}>0(k=1, \ldots, n)$ be real numbers such that $P_{k}=\sum_{i=1}^{k} p_{i}(k=1, \ldots, n)$. Let $P_{k-1}, P_{k}, \frac{\sum_{i=1}^{k-1} p_{i} a_{i}}{a_{k}}$ and $\frac{\sum_{i=1}^{k} p_{i} a_{i}}{a_{k}} \in$ $[a, b]$ for all $k=2, \ldots, n$ and $g:[a, b] \rightarrow \mathbb{R}$ be a differentiable function such that $g(x+h)-g(x)$ is convex for all $x, x+h \in[a, b]$, where $h \geq 0$. Then for any $s \in \mathbb{R}$, we have

$$
\begin{aligned}
\sum_{k=2}^{n} a_{k}^{s}\left(g\left(P_{k}\right)-g\left(P_{k-1}\right)\right) & \\
+ & \sum_{k=2}^{n} a_{k}^{s-1}\left(\sum_{i=1}^{k-1} p_{i} a_{i}-P_{k-1} a_{k}\right)\left(g^{\prime}\left(P_{k}\right)-g^{\prime}\left(P_{k-1}\right)\right) \\
& \leq \sum_{k=2}^{n} a_{k}^{s}\left(g\left(\frac{\sum_{i=1}^{k} p_{i} a_{i}}{a_{k}}\right)-g\left(\frac{\sum_{i=1}^{k-1} p_{i} a_{i}}{a_{k}}\right)\right) \\
\leq & \sum_{k=2}^{n} a_{k}^{s}\left(g\left(P_{k}\right)-g\left(P_{k-1}\right)\right)+\sum_{k=2}^{n} a_{k}^{s-1}\left(\sum_{i=1}^{k-1} p_{i} a_{i}-P_{k-1} a_{k}\right) . \\
& \cdot\left(g^{\prime}\left(\frac{\sum_{i=1}^{k} p_{i} a_{i}}{a_{k}}\right)-g^{\prime}\left(\frac{\sum_{i=1}^{k-1} p_{i} a_{i}}{a_{k}}\right)\right) .
\end{aligned}
$$

If $g(x+h)-g(x)$ is concave for all $x, x+h \in[a, b]$ such that $h \geq 0$, then opposite inequalities hold in (2.1).

Proof. Since $g(x+h)-g(x)$ is a convex function, where $g$ is differentiable, by setting $f(x)=g(x+h)-g(x)$ in (1.11), we have

$$
\begin{aligned}
g(y+ & h)-g(y)+(x-y)\left(g^{\prime}(y+h)-g^{\prime}(y)\right) \\
& \leq g(x+h)-g(x) \\
& \leq g(y+h)-g(y)+(x-y)\left(g^{\prime}(x+h)-g^{\prime}(x)\right) .
\end{aligned}
$$


Substituting $x=\frac{\sum_{i=1}^{k-1} p_{i} a_{i}}{a_{k}}, y=P_{k-1}$ and $h=p_{k}(k=2, \ldots, n)$, where $a_{k}>0$ $(k=1, \ldots, n)$ in $(2.2)$, we have,

$$
\begin{aligned}
g\left(P_{k}\right)- & g\left(P_{k-1}\right)+\left(\frac{\sum_{i=1}^{k-1} p_{i} a_{i}-P_{k-1} a_{k}}{a_{k}}\right)\left(g^{\prime}\left(P_{k}\right)-g^{\prime}\left(P_{k-1}\right)\right) \\
\leq & g\left(\frac{\sum_{i=1}^{k} p_{i} a_{i}}{a_{k}}\right)-g\left(\frac{\sum_{i=1}^{k-1} p_{i} a_{i}}{a_{k}}\right) \\
\leq & g\left(P_{k}\right)-g\left(P_{k-1}\right)+\left(\frac{\sum_{i=1}^{k-1} p_{i} a_{i}-P_{k-1} a_{k}}{a_{k}}\right) . \\
& \cdot\left(g^{\prime}\left(\frac{\sum_{i=1}^{k} p_{i} a_{i}}{a_{k}}\right)-g^{\prime}\left(\frac{\sum_{i=1}^{k-1} p_{i} a_{i}}{a_{k}}\right)\right) .
\end{aligned}
$$

Multiplying (2.3) throughout by $a_{k}^{s}>0(k=1, \ldots, n)$, where $s \in \mathbb{R}$ and summing over $k$ from 2 to $n$, we have $(2.1)$.

If $g(x+h)-g(x)$ is concave for all $x, x+h \in[a, b]$ such that $h \geq 0$, then we have opposite inequalities in (1.11) and in the same way we have opposite inequalities in (2.1).

Corollary 2.2. Let $a_{k}>0$ and $p_{k}>0(k=1, \ldots, n)$ be real numbers such that $P_{k}=\sum_{i=1}^{k} p_{i}(k=1, \ldots, n)$. Let $P_{k-1}, P_{k}, \frac{\sum_{i=1}^{k-1} p_{i} a_{i}}{a_{k}}$ and $\frac{\sum_{i=1}^{k} p_{i} a_{i}}{a_{k}} \in[a, b]$ for all $k=2, \ldots, n$ and $g:[a, b] \rightarrow \mathbb{R}$ be a differentiable function. If the function $g$ is 3-convex, then for any $s \in \mathbb{R}(2.1)$ holds and if it is 3-concave, then (2.1) holds in the reverse direction.

Proof. Since $g$ is a 3 -convex function, $g^{\prime}$ exists and is convex on $[a, b]$ (see $[8, \mathrm{p} .16]$ ). Therefore for any $h \geq 0$ such that $x, x+h \in[a, b], f^{\prime}(x)$ exists, where $f(x):=g(x+h)-g(x)$ and we can write

$$
\frac{f^{\prime}(y)-f^{\prime}(x)}{y-x}=\frac{g^{\prime}(y+h)-g^{\prime}(x+h)}{(y+h)-(x+h)}-\frac{g^{\prime}(y)-g^{\prime}(x)}{y-x} .
$$

Now by using Proposition 1.9, we have $\frac{f^{\prime}(y)-f^{\prime}(x)}{y-x} \geq 0$, showing that $f^{\prime}$ is nondecreasing on $[a, b]$ and so $f$ is convex. By taking $f(x)=g(x+h)-g(x)$ in (1.11) and by making the same substitutions as given in the proof of Theorem 2.1 , we have (2.1).

Similarly, if $g$ is a 3 -concave function, then it is easy to prove that $f$ is concave and so we then have opposite inequalities in (1.11) and in the same way we have opposite inequalities in (2.1).

The second main theorem is again the generalization of Theorems 1.4 and 1.5.

THEOREM 2.3. Let $a_{k}>0$ and $p_{k}>0(k=1, \ldots, n)$ be real numbers such that $P_{k}=\sum_{i=1}^{k} p_{i}(k=1, \ldots, n)$. Let $p_{1} a_{1}, P_{k-1} a_{k}, P_{k} a_{k}, \sum_{i=1}^{k-1} p_{i} a_{i}$ 
and $\sum_{i=1}^{k} p_{i} a_{i} \in[a, b]$ for all $k=2, \ldots, n$ and $g:[a, b] \rightarrow \mathbb{R}$ be a differentiable function such that $g(x+h)-g(x)$ is convex for all $x, x+h \in[a, b]$, where $h \geq 0$. Then we have,

$$
\begin{aligned}
g\left(p_{1} a_{1}\right) & +\sum_{k=2}^{n}\left(g\left(P_{k} a_{k}\right)-g\left(P_{k-1} a_{k}\right)\right) \\
+ & \sum_{k=2}^{n}\left(\sum_{i=1}^{k-1} p_{i} a_{i}-P_{k-1} a_{k}\right)\left(g^{\prime}\left(P_{k} a_{k}\right)-g^{\prime}\left(P_{k-1} a_{k}\right)\right) \\
\leq & g\left(\sum_{i=1}^{n} p_{i} a_{i}\right) \\
\leq & g\left(p_{1} a_{1}\right)+\sum_{k=2}^{n}\left(g\left(P_{k} a_{k}\right)-g\left(P_{k-1} a_{k}\right)\right) \\
& +\sum_{k=2}^{n}\left(\sum_{i=1}^{k-1} p_{i} a_{i}-P_{k-1} a_{k}\right)\left(g^{\prime}\left(\sum_{i=1}^{k} p_{i} a_{i}\right)-g^{\prime}\left(\sum_{i=1}^{k-1} p_{i} a_{i}\right)\right) .
\end{aligned}
$$

If $g(x+h)-g(x)$ is concave for all $x, x+h \in[a, b]$ such that $h \geq 0$, then opposite inequalities hold in (2.4).

Proof. Since $g(x+h)-g(x)$ is a convex function, where $g$ is differentiable, by setting $f(x)=g(x+h)-g(x)$ in (1.11), we obtain (2.2). Substituting $x=\sum_{i=1}^{k-1} p_{i} a_{i}, y=P_{k-1} a_{k}$ and $h=p_{k} a_{k}(k=2, \ldots, n)$ in (2.2), where $a_{k}>0(k=1, \ldots, n)$, we get

$$
\begin{aligned}
g\left(P_{k} a_{k}\right) & -g\left(P_{k-1} a_{k}\right)+\left(\sum_{i=1}^{k-1} p_{i} a_{i}-P_{k-1} a_{k}\right)\left(g^{\prime}\left(P_{k} a_{k}\right)-g^{\prime}\left(P_{k-1} a_{k}\right)\right) \\
\leq & g\left(\sum_{i=1}^{k} p_{i} a_{i}\right)-g\left(\sum_{i=1}^{k-1} p_{i} a_{i}\right) \leq g\left(P_{k} a_{k}\right)-g\left(P_{k-1} a_{k}\right) \\
& +\left(\sum_{i=1}^{k-1} p_{i} a_{i}-P_{k-1} a_{k}\right)\left(g^{\prime}\left(\sum_{i=1}^{k} p_{i} a_{i}\right)-g^{\prime}\left(\sum_{i=1}^{k-1} p_{i} a_{i}\right)\right) .
\end{aligned}
$$

Summing over $k$ from 2 to $n$, we obtain (2.4).

If $g(x+h)-g(x)$ is concave for all $x, x+h \in[a, b]$ such that $h \geq 0$, then we have opposite inequalities in (1.11) and in the same way we have opposite inequalities in (2.4).

Corollary 2.4. Let $a_{k}>0$ and $p_{k}>0(k=1, \ldots, n)$ be real numbers such that $P_{k}=\sum_{i=1}^{k} p_{i}(k=1, \ldots, n)$. Let $p_{1} a_{1}, P_{k-1} a_{k}, P_{k} a_{k}, \sum_{i=1}^{k-1} p_{i} a_{i}$ and $\sum_{i=1}^{k} p_{i} a_{i} \in[a, b]$ for all $k=2, \ldots, n$ and $g:[a, b] \rightarrow \mathbb{R}$ be a differentiable function. If the function $g$ is 3-convex, then (2.4) holds and if it is 3-concave, then (2.4) holds in the reverse direction. 
Proof. Analogous to the proof of Corollary 2.2.

REMARK 2.5. For $g(x)=x^{s}$, the function $g(x+h)-g(x)$ is convex when $0<s<1$ or $s>2$ and concave when $1<s<2$ on $(0, \infty)$ with $h \geq 0$. The results from Theorem 1.4 can be recaptured by making the substitutions $p_{k} \rightarrow 1(k=1, \ldots, n)$ and $g(x)=x^{s}, x \in(0, \infty)$ in the results of Theorems 2.1 and 2.3 and in this way these theorems are the generalizations of Theorem 1.4. It is easy to see that $g(x+h)-g(x)$ is convex for $g(x)=-x \ln x$ and concave for $g(x)=x \ln x$ on $(0, \infty)$ with $h \geq 0$. If we make the substitutions $p_{k} \rightarrow 1(k=1, \ldots, n)$ in Theorems 2.1 and 2.3 and then replace $a_{k} \rightarrow p_{k}$ $(k=1, \ldots, n)$ and take $g(x)=-x \ln x$ in (2.1) and (2.4) or $g(x)=x \ln x$ in their reverses and also using the fact that

$$
P_{n}=\sum_{i=1}^{n} p_{i}=1
$$

then we (1.6) follows. In this way Theorems 2.1 and 2.3 are the generalizations of the Theorem 1.5.

REMARK 2.6. It is easy to see that the function $g(x)=x^{s}$, where $x \in$ $(0, \infty)$ is both convex and 3-convex for $s>2$, convex and 3-concave for $1<$ $s<2$ and concave and 3 -convex for $0<s<1$. If we make the substitutions $p_{k} \rightarrow 1(k=1, \ldots, n)$ and $g(x)=x^{s}, x \in(0, \infty)$ in Corollary 2.2 or Corollary 2.4 and if $\left(a_{k}>0, k=1, \ldots, n\right) \subset \mathbb{R}$ is a sequence non-increasing in mean, then the left inequality in (2.1) and (2.4) is a refinement of (1.2) for $s>2$, and the right inequality in (2.1) and (2.4) is a refinement of the reversed inequality of (1.2) for $0<s<1$. In case of 3 -concave functions, the right inequality in the reverse of (2.1) and (2.4) is a refinement of (1.2) for $1<s<2$.

More generally, if a sequence $\left(a_{k}>0, k=1, \ldots, n\right) \subset \mathbb{R}$ is non-increasing in weighted mean and the function $g$ is convex and 3-convex, then the left inequality in (2.4) is a refinement of (1.1). If $g$ is convex and 3-concave, then the inequalities in (2.4) are reversed and the right inequality is the refinement of (1.1). Analogous statements can be made for a function which is concave and 3-convex or concave and 3-concave.

Let us define functionals $\Phi_{i}(i=1, \ldots, 6)$ by the non-negative differences of the inequalities (2.1) and (2.4) as follows:

$$
\begin{aligned}
\Phi_{1}(g)= & \sum_{k=2}^{n} a_{k}^{s}\left(g\left(\frac{\sum_{i=1}^{k} p_{i} a_{i}}{a_{k}}\right)-g\left(\frac{\sum_{i=1}^{k-1} p_{i} a_{i}}{a_{k}}\right)-\left(g\left(P_{k}\right)-g\left(P_{k-1}\right)\right)\right) \\
& -\sum_{k=2}^{n} a_{k}^{s-1}\left(\sum_{i=1}^{k-1} p_{i} a_{i}-P_{k-1} a_{k}\right)\left(g^{\prime}\left(P_{k}\right)-g^{\prime}\left(P_{k-1}\right)\right),
\end{aligned}
$$




$$
\begin{aligned}
\Phi_{2}(g)= & \sum_{k=2}^{n} a_{k}^{s-1}\left(\sum_{i=1}^{k-1} p_{i} a_{i}-P_{k-1} a_{k}\right)\left(g^{\prime}\left(\frac{\sum_{i=1}^{k} p_{i} a_{i}}{a_{k}}\right)-g^{\prime}\left(\frac{\sum_{i=1}^{k-1} p_{i} a_{i}}{a_{k}}\right)\right) \\
& -\sum_{k=2}^{n} a_{k}^{s-1}\left(\sum_{i=1}^{k-1} p_{i} a_{i}-P_{k-1} a_{k}\right)\left(g^{\prime}\left(P_{k}\right)-g^{\prime}\left(P_{k-1}\right)\right)
\end{aligned}
$$

$\Phi_{3}(g)=\sum_{k=2}^{n} a_{k}^{s}\left(g\left(P_{k}\right)-g\left(P_{k-1}\right)-\left(g\left(\frac{\sum_{i=1}^{k} p_{i} a_{i}}{a_{k}}\right)-g\left(\frac{\sum_{i=1}^{k-1} p_{i} a_{i}}{a_{k}}\right)\right)\right)$

$$
+\sum_{k=2}^{n} a_{k}^{s-1}\left(\sum_{i=1}^{k-1} p_{i} a_{i}-P_{k-1} a_{k}\right)\left(g^{\prime}\left(\frac{\sum_{i=1}^{k} p_{i} a_{i}}{a_{k}}\right)-g^{\prime}\left(\frac{\sum_{i=1}^{k-1} p_{i} a_{i}}{a_{k}}\right)\right),
$$

$$
\begin{aligned}
\Phi_{4}(g)= & g\left(\sum_{i=1}^{n} p_{i} a_{i}\right)-g\left(p_{1} a_{1}\right)-\sum_{k=2}^{n}\left(g\left(P_{k} a_{k}\right)-g\left(P_{k-1} a_{k}\right)\right) \\
& -\sum_{k=2}^{n}\left(\sum_{i=1}^{k-1} p_{i} a_{i}-P_{k-1} a_{k}\right)\left(g^{\prime}\left(P_{k} a_{k}\right)-g^{\prime}\left(P_{k-1} a_{k}\right)\right),
\end{aligned}
$$

$$
\begin{aligned}
\Phi_{5}(g)= & \sum_{k=2}^{n}\left(\sum_{i=1}^{k-1} p_{i} a_{i}-P_{k-1} a_{k}\right)\left(g^{\prime}\left(\sum_{i=1}^{k} p_{i} a_{i}\right)-g^{\prime}\left(\sum_{i=1}^{k-1} p_{i} a_{i}\right)\right) \\
& -\sum_{k=2}^{n}\left(\sum_{i=1}^{k-1} p_{i} a_{i}-P_{k-1} a_{k}\right)\left(g^{\prime}\left(P_{k} a_{k}\right)-g^{\prime}\left(P_{k-1} a_{k}\right)\right)
\end{aligned}
$$

and

$(2.10)$

$$
\begin{aligned}
\Phi_{6}(g)= & g\left(p_{1} a_{1}\right)-g\left(\sum_{i=1}^{n} p_{i} a_{i}\right)+\sum_{k=2}^{n}\left(g\left(P_{k} a_{k}\right)-g\left(P_{k-1} a_{k}\right)\right) \\
& +\sum_{k=2}^{n}\left(\sum_{i=1}^{k-1} p_{i} a_{i}-P_{k-1} a_{k}\right)\left(g^{\prime}\left(\sum_{i=1}^{k} p_{i} a_{i}\right)-g^{\prime}\left(\sum_{i=1}^{k-1} p_{i} a_{i}\right)\right),
\end{aligned}
$$

where

$$
P_{k-1}, P_{k}, P_{k-1} a_{k}, P_{k} a_{k}, \sum_{i=1}^{k-1} p_{i} a_{i}, \sum_{i=1}^{k} p_{i} a_{i}, \frac{\sum_{i=1}^{k-1} p_{i} a_{i}}{a_{k}}, \frac{\sum_{i=1}^{k} p_{i} a_{i}}{a_{k}} \in[a, b]
$$

are such that $a_{k}>0, p_{k}>0(k=1, \ldots, n)$ with $P_{k-1}=\sum_{i=1}^{k-1} p_{i} a_{i}$ for all $k=2, \ldots, n$. If the function $g$ is differentiable and 3 -convex defined on $[a, b]$, then Corollaries 2.2 and 2.4 imply that

$$
\Phi_{i}(g) \geq 0, \quad i=1, \ldots, 6 .
$$


Now, we give mean value theorems for the functionals $\Phi_{i}(i=1, \ldots, 6)$ as defined in $(2.5)-(2.10)$. These theorems enable us to define various classes of means that can be expressed in terms of linear functionals.

TheOREM 2.7. Let $a_{k}>0$ and $p_{k}>0(k=1, \ldots, n)$ be real numbers such that $P_{k}=\sum_{i=1}^{k} p_{i}(k=1, \ldots, n)$. Let $P_{k-1}, P_{k}, P_{k-1} a_{k}, P_{k} a_{k}, \sum_{i=1}^{k-1} p_{i} a_{i}$, $\sum_{i=1}^{k} p_{i} a_{i}, \frac{\sum_{i=1}^{k-1} p_{i} a_{i}}{a_{k}}$ and $\frac{\sum_{i=1}^{k} p_{i} a_{i}}{a_{k}} \in[a, b]$ for all $k=2, \ldots, n$. Suppose that $\Phi_{i}(i=1, \ldots, 6)$ are linear functionals defined as in $(2.5)-(2.10)$ and $g \in$ $C^{3}([a, b])$. Then there exists $\xi_{i} \in[a, b]$ such that

$$
\Phi_{i}(g)=\frac{g^{\prime \prime \prime}\left(\xi_{i}\right)}{6} \Phi_{i}\left(g_{0}\right), \quad i=1, \ldots, 6,
$$

where $g_{0}(x)=x^{3}$.

Proof. Since $g^{\prime \prime \prime}(x)$ is continuous on $[a, b]$, there exist real numbers $m=$ $\min _{x \in[a, b]} g^{\prime \prime \prime}(x)$ and $M=\max _{x \in[a, b]} g^{\prime \prime \prime}(x)$ such that $m \leq g^{\prime \prime \prime}(x) \leq M$. It is easy to see that the functions $g_{1}(x)$ and $g_{2}(x)$ defined by $g_{1}(x)=\frac{M x^{3}}{6}-g(x)$ and $g_{2}(x)=g(x)-\frac{m x^{3}}{6}$ are 3 -convex. Therefore, by using $g_{1}$ and $g_{2}$ in (2.11), we have $\Phi_{i}\left(\frac{M x^{3}}{6}-g(x)\right) \geq 0$ and $\Phi_{i}\left(g(x)-\frac{m x^{3}}{6}\right) \geq 0(i=1, \ldots, 6)$, which are equivalent to

$$
\Phi_{i}(g) \leq \frac{M}{6} \Phi_{i}\left(g_{0}\right), \quad i=1, \ldots, 6
$$

and

$$
\Phi_{i}(g) \geq \frac{m}{6} \Phi_{i}\left(g_{0}\right), \quad i=1, \ldots, 6,
$$

respectively. From (2.13) and (2.14), we have

$$
\frac{m}{6} \Phi_{i}\left(g_{0}\right) \leq \Phi_{i}(g) \leq \frac{M}{6} \Phi_{i}\left(g_{0}\right), \quad i=1, \ldots, 6 .
$$

If $\Phi_{i}\left(g_{0}\right)=0(i=1, \ldots, 6)$, then there is nothing to prove. Let $\Phi_{i}\left(g_{0}\right)>0$ $(i=1, \ldots, 6)$, then from $(2.15)$, we have

$$
m \leq \frac{6 \Phi_{i}(g)}{\Phi_{i}\left(g_{0}\right)} \leq M, \quad i=1, \ldots, 6 .
$$

Now, by using the fact that for $m \leq \eta_{i} \leq M$, there exist $\xi_{i} \in[a, b]$ such that $g^{\prime \prime \prime}\left(\xi_{i}\right)=\eta_{i}(i=1, \ldots, 6)$, and so we have $(2.12)$.

TheOREM 2.8. Let all the conditions of Theorem 2.7 are satisfied. Then there exists $\xi_{i} \in[a, b]$ such that

$$
\frac{\Phi_{i}(g)}{\Phi_{i}(h)}=\frac{g^{\prime \prime \prime}\left(\xi_{i}\right)}{h^{\prime \prime \prime}\left(\xi_{i}\right)}, \quad i=1, \ldots, 6,
$$

provided that the denominators are non-zero. 
Proof. Consider the function $\psi_{i} \in C^{3}([a, b])$ defined by $\psi_{i}=c_{i} g-d_{i} h$, where $c_{i}=\Phi_{i}(h)$ and $d_{i}=\Phi_{i}(g)(i=1, \ldots, 6)$. Using Theorem 2.7 with $g=\psi_{i}$, there exist $\xi_{i} \in[a, b]$ such that

$$
\left(\frac{c_{i} g^{\prime \prime \prime}\left(\xi_{i}\right)}{6}-\frac{d_{i} h^{\prime \prime \prime}\left(\xi_{i}\right)}{6}\right) \Phi_{i}\left(g_{0}\right)=0, \quad i=1, \ldots, 6 .
$$

Since $\Phi_{i}\left(g_{0}\right) \neq 0$ (because otherwise we have a contradiction with $\Phi_{i}(h) \neq 0$ by Theorem 2.7), we get

$$
\frac{g^{\prime \prime \prime}\left(\xi_{i}\right)}{h^{\prime \prime \prime}\left(\xi_{i}\right)}=\frac{d_{i}}{c_{i}}, \quad i=1, \ldots, 6 .
$$

After substituting the values of $c_{i}$ and $d_{i}$, we have (2.16).

REMARK 2.9. (i) By taking $g(x)=x^{s}$ and $h(x)=x^{q}$ in (2.16), where $s, q \in \mathbb{R} \backslash\{0,1,2\}$ are such that $s \neq q$, we have

$$
\xi_{i}^{s-q}=\frac{q(q-1)(q-2) \Phi_{i}\left(x^{s}\right)}{s(s-1)(s-2) \Phi_{i}\left(x^{q}\right)}, \quad i=1, \ldots, 6 .
$$

(ii) If the inverse of the function $g^{\prime \prime \prime} / h^{\prime \prime \prime}$ exists, then (2.16) gives

$$
\xi_{i}=\left(\frac{g^{\prime \prime \prime}}{h^{\prime \prime \prime}}\right)^{-1}\left(\frac{\Phi_{i}(g)}{\Phi_{i}(h)}\right), \quad i=1, \ldots, 6 .
$$

3. $n$-Exponential Convexity And Log-Convexity Of The Functions Associated With The Differences Of The Generalized Inequalities

We begin this section by recollecting the definitions and properties which are going to be explored here and also some useful characterizations of these properties. In the sequel, let $I$ be an open interval in $\mathbb{R}$.

Definition 3.1. A function $h: I \rightarrow \mathbb{R}$ is n-exponentially convex in the Jensen sense on $I$ if

$$
\sum_{i, j=1}^{n} \alpha_{i} \alpha_{j} h\left(\frac{x_{i}+x_{j}}{2}\right) \geq 0
$$

holds for every $\alpha_{i} \in \mathbb{R}$ and $x_{i} \in I, i=1, \ldots, n$ (see [10]).

Definition 3.2. A function $h: I \rightarrow \mathbb{R}$ is n-exponentially convex on $I$ if it is n-exponentially convex in the Jensen sense and continuous on $I$.

Remark 3.3. From the above definition, it is clear that 1-exponentially convex functions in the Jensen sense are non-negative functions. Also, $n$ exponentially convex functions in the Jensen sense are k-exponentially convex functions in the Jensen sense for all $k \in \mathbb{N}, k \leq n$. 
Positive semi-definite matrices represent a basic tool in our study. By the definition of positive semi-definite matrices and some basic linear algebra, we have the following proposition.

Proposition 3.4. If $h$ is n-exponentially convex in the Jensen sense, then the matrix $\left[h\left(\frac{x_{i}+x_{j}}{2}\right)\right]_{i, j=1}^{k}$ is a positive semi-definite matrix for all $k \in \mathbb{N}, k \leq n$. In particular

$\operatorname{det}\left[h\left(\frac{x_{i}+x_{j}}{2}\right)\right]_{i, j=1}^{k} \geq 0 \quad$ for every $k \in \mathbb{N}, k \leq n, \quad x_{i} \in I, i=1, \ldots, n$.

Definition 3.5. A function $h: I \rightarrow \mathbb{R}$ is exponentially convex in the Jensen sense if it is n-exponentially convex in the Jensen sense for all $n \in \mathbb{N}$.

Definition 3.6. A function $h: I \rightarrow \mathbb{R}$ is exponentially convex if it is exponentially convex in the Jensen sense and continuous.

Lemma 3.7. A function $h: I \rightarrow(0, \infty)$ is $\log$-convex in the Jensen sense, that is, for every $x, y \in I$,

$$
h^{2}\left(\frac{x+y}{2}\right) \leq h(x) h(y)
$$

holds if and only if the relation

$$
\alpha^{2} h(x)+2 \alpha \beta h\left(\frac{x+y}{2}\right)+\beta^{2} h(y) \geq 0
$$

holds for every $\alpha, \beta \in \mathbb{R}$ and $x, y \in I$.

REMARK 3.8. It follows that a function is log-convex in the Jensen-sense if and only if it is 2-exponentially convex in the Jensen sense. Also, by using the basic convexity theory, a function is log-convex if and only if it is 2exponentially convex. For more results about log-convexity see [1] and the references therein.

Proposition 3.9. A 3rd-order divided difference of a function $f:[a, b] \rightarrow$ $\mathbb{R}$ at the points $y_{0}, y_{1}, y_{2}, y_{3} \in[a, b]$ can be expressed in the following forms:

(i) If $y_{0}, y_{1}, y_{2}, y_{3} \in[a, b]$ such that $y_{i} \neq y_{j}, i \neq j, i, j=0, \ldots 3$, then we have

$$
\left[y_{0}, y_{1}, y_{2}, y_{3} ; f\right]=\sum_{i=0}^{3} \frac{f\left(y_{i}\right)}{q^{\prime}\left(y_{i}\right)}, \quad \text { where } q(y)=\prod_{j=0}^{3}\left(y-y_{j}\right) .
$$


(ii) If $f$ is a differentiable function defined on $[a, b]$ and $y, y_{0}, y_{1} \in[a, b]$ such that $y \neq y_{0} \neq y_{1}$, then we have

$$
\begin{aligned}
{\left[y, y, y_{0}, y_{1} ; f\right]=} & \frac{f^{\prime}(y)}{\left(y-y_{0}\right)\left(y-y_{1}\right)}+\frac{f(y)\left(y_{0}+y_{1}-2 y\right)}{\left(y-y_{0}\right)^{2}\left(y-y_{1}\right)^{2}} \\
& +\frac{f\left(y_{0}\right)}{\left(y_{0}-y\right)^{2}\left(y_{0}-y_{1}\right)}+\frac{f\left(y_{1}\right)}{\left(y_{1}-y\right)^{2}\left(y_{1}-y_{0}\right)} .
\end{aligned}
$$

(iii) If $f$ is a differentiable function defined on $[a, b]$ and $y, y_{0} \in[a, b]$ such that $y \neq y_{0}$, then we have

$$
\left[y, y, y_{0}, y_{0} ; f\right]=\frac{\left(y_{0}-y\right)\left(f^{\prime}\left(y_{0}\right)+f^{\prime}(y)\right)+2\left(f(y)-f\left(y_{0}\right)\right)}{\left(y_{0}-y\right)^{3}} .
$$

(iv) If $f$ is twice differentiable function defined on $[a, b]$ and $y, y_{0} \in[a, b]$ such that $y \neq y_{0}$, then we have

$$
\left[y, y, y, y_{0} ; f\right]=\frac{1}{\left(y_{0}-y\right)^{3}}\left[f\left(y_{0}\right)-\sum_{i=0}^{2} \frac{f^{(i)}(y)}{i !}\left(y_{0}-y\right)^{i}\right] .
$$

(v) If $f$ is three times differentiable function defined on $[a, b]$ and $y \in[a, b]$, then we have

$$
[y, y, y, y ; f]=\frac{f^{\prime \prime \prime}(y)}{3 !} .
$$

The following theorem shows that the definition of 3-convex function can be extended by including the cases in which some or all the points coincide.

TheOREM 3.10. Let $f$ be a function defined on $[a, b] \subset \mathbb{R}$, then the following statements hold.

(i) If $f \in C^{1}([a, b])$, then $f$ is 3 -convex if and only if $\left[y, y, y_{0}, y_{1} ; f\right] \geq 0$ for all $y \neq y_{0} \neq y_{1}$ in $[a, b]$.

(ii) If $f \in C^{1}([a, b])$, then $f$ is 3 -convex if and only if $[y, y, z, z ; f] \geq 0$ for all $y \neq z$ in $[a, b]$.

(iii) If $f \in C^{2}([a, b])$, then $f$ is 3 -convex if and only if $\left[y, y, y, y_{0} ; f\right] \geq 0$ for all $y \neq y_{0}$ in $[a, b]$.

(iv) If $f \in C^{3}([a, b])$, then $f$ is 3 -convex if and only if $[y, y, y, y ; f] \geq 0$ for all $y \in[a, b]$.

PRoOF. It can be proved easily by using the mean value theorems for divided differences (see [4]).

Next, we study the $n$-exponential convexity and log-convexity of the functions associated with the linear functionals $\Phi_{i}(i=1, \ldots, 6)$ defined in $(2.5)-(2.10)$.

THEOREM 3.11. Let $\Omega=\left\{f_{s}: s \in I \subseteq \mathbb{R}\right\}$ be a family of differentiable functions defined on $[a, b]$ such that the function $s \mapsto\left[y_{0}, y_{1}, y_{2}, y_{3} ; f_{s}\right]$ is $n$ exponentially convex in the Jensen sense on I for every four mutually distinct 
points $y_{0}, y_{1}, y_{2} \cdot y_{3} \in[a, b]$. Let $\Phi_{i}(i=1, \ldots, 6)$ be linear functionals defined as in (2.5)-(2.10). Then the following statements hold.

(i) The function $s \mapsto \Phi_{i}\left(f_{s}\right)$ is n-exponentially convex in the Jensen sense on $I$ and the matrix $\left[\Phi_{i}\left(f_{\frac{s_{j}+s_{k}}{2}}\right)\right]_{j, k=1}^{m}$ is a positive semi-definite matrix for all $m \in \mathbb{N}, m \leq n$ and $s_{1}, \ldots, s_{m} \in I$. Particularly,

$$
\operatorname{det}\left[\Phi_{i}\left(f_{\frac{s_{j}+s_{k}}{2}}\right)\right]_{j, k=1}^{m} \geq 0, \forall m \in \mathbb{N}, m \leq n .
$$

(ii) If the function $s \mapsto \Phi_{i}\left(f_{s}\right)$ is continuous on $I$, then it is n-exponentially convex on $I$.

Proof. The idea of the proof is the same as that of Theorem 3.1 in [10].

(i) Let $\alpha_{j} \in \mathbb{R}(j=1, \ldots, n)$ and consider the function

$$
\varphi(y)=\sum_{j, k=1}^{n} \alpha_{j} \alpha_{k} f_{\frac{s_{j}+s_{k}}{2}}(y),
$$

where $s_{j} \in I$ and $f_{\frac{s_{j}+s_{k}}{2}} \in \Omega$. Then

$$
\left[y_{0}, y_{1}, y_{2}, y_{3} ; \varphi\right]=\sum_{j, k=1}^{n} \alpha_{j} \alpha_{k}\left[y_{0}, y_{1}, y_{2}, y_{3} ; \frac{f_{s_{j}+s_{k}}}{2}\right]
$$

and since $\left[y_{0}, y_{1}, y_{2}, y_{3} ; \frac{f_{s_{j}+s_{k}}}{2}\right]$ is $n$-exponentially convex in the Jensen sense on $I$ by assumption, it follows that

$$
\left[y_{0}, y_{1}, y_{2}, y_{3} ; \varphi\right]=\sum_{j, k=1}^{n} \alpha_{j} \alpha_{k}\left[y_{0}, y_{1}, y_{2}, y_{3} ; \frac{f_{s_{j}+s_{k}}}{2}\right] \geq 0
$$

And so, by using Definition 1.11 for $n=3$, we conclude that $\varphi$ is a 3-convex function. Hence

$$
\Phi_{i}(\varphi) \geq 0, \quad i=1, \ldots, 6,
$$

which is equivalent to

$$
\sum_{j, k=1}^{n} \alpha_{j} \alpha_{k} \Phi_{i}\left(f_{\frac{s_{j}+s_{k}}{2}}\right) \geq 0, \quad i=1, \ldots, 6,
$$

and so we conclude that the function $s \mapsto \Phi_{i}\left(f_{s}\right)$ is $n$-exponentially convex in the Jensen sense on $I$.

The remaining part follows from Proposition 3.4.

(ii) If the function $s \mapsto \Phi_{i}\left(f_{s}\right)$ is continuous on $I$, then from (i) and by Definition 3.2 it follows that it is $n$-exponentially convex on $I$. 
The following corollary is an immediate consequence of the above theorem.

Corollary 3.12. Let $\Omega=\left\{f_{s}: s \in I \subseteq \mathbb{R}\right\}$ be a family of differentiable functions defined on $[a, b]$ such that the function $s \mapsto\left[y_{0}, y_{1}, y_{2}, y_{3} ; f_{s}\right]$ is exponentially convex in the Jensen sense on I for every four mutually distinct points $y_{0}, y_{1}, y_{2}, y_{3} \in[a, b]$. Let $\Phi_{i}(i=1, \ldots, 6)$ be linear functionals defined as in (2.5)-(2.10). Then the following statements hold.

(i) The function $s \mapsto \Phi_{i}\left(f_{s}\right)$ is exponentially convex in the Jensen sense on $I$ and the matrix $\left[\Phi_{i}\left(f_{\frac{s_{j}+s_{k}}{2}}\right)\right]_{j, k=1}^{n}$ is a positive semi-definite matrix for all $n \in \mathbb{N}$ and $s_{1}, . ., s_{n} \in I$. Particularly,

$$
\operatorname{det}\left[\Phi_{i}\left(f_{\frac{s_{j}+s_{k}}{2}}\right)\right]_{j, k=1}^{n} \geq 0, \forall n \in \mathbb{N} .
$$

(ii) If the function $s \mapsto \Phi_{i}\left(f_{s}\right)$ is continuous on $I$, then it is exponentially convex on $I$.

Corollary 3.13. Let $\Omega=\left\{f_{s}: s \in I \subseteq \mathbb{R}\right\}$ be a family of differentiable functions defined on $[a, b]$ such that the function $s \mapsto\left[y_{0}, y_{1}, y_{2}, y_{3} ; f_{s}\right]$ is 2exponentially convex in the Jensen sense on I for every four mutually distinct points $y_{0}, y_{1}, y_{2}, y_{3} \in[a, b]$. Let $\Phi_{i}(i=1, \ldots, 6)$ be linear functionals defined as in (2.5)-(2.10). Further, assume that $\Phi_{i}\left(f_{s}\right)(i=1, \ldots, 6)$ is strictly positive for $f_{s} \in \Omega$. Then the following statements hold.

(i) If the function $s \mapsto \Phi_{i}\left(f_{s}\right)$ is continuous on $I$, then it is 2-exponentially convex on $I$ and so it is log-convex on $I$ and for $r, s, t \in I$ such that $r<t<s$, we have

$$
\left[\Phi_{i}\left(f_{t}\right)\right]^{s-r} \leq\left[\Phi_{i}\left(f_{r}\right)\right]^{s-t}\left[\Phi_{i}\left(f_{s}\right)\right]^{t-r},
$$

known as Lyapunov's inequality. If $r<s<t$ or $t<r<s$, then opposite inequalities hold in (3.1).

(ii) If the function $s \mapsto \Phi_{i}\left(f_{s}\right)$ is differentiable on $I$, then for every $s, q, u, v \in I$ such that $s \leq u$ and $q \leq v$, we have

$$
\mu_{s, q}\left(\Phi_{i}, \Omega\right) \leq \mu_{u, v}\left(\Phi_{i}, \Omega\right)
$$

where

$$
\mu_{s, q}\left(\Phi_{i}, \Omega\right)=\left\{\begin{array}{cc}
\left(\frac{\Phi_{i}\left(f_{s}\right)}{\Phi_{i}\left(f_{q}\right)}\right)^{\frac{1}{s-q}}, & s \neq q, \\
\exp \left(\frac{\frac{d}{d s} \Phi_{i}\left(f_{s}\right)}{\Phi_{i}\left(f_{s}\right)}\right), & s=q,
\end{array}\right.
$$

for $f_{s}, f_{q} \in \Omega$.

Proof. The idea of the proof is the same as that in the proof of $[10$, Corollary 3.2]. 
(i) The claim that the function $s \mapsto \Phi_{i}\left(f_{s}\right)$ is log-convex on $I$ is an immediate consequence of Theorem 3.11 and Remark 3.8, and (3.1) can be obtained by replacing the convex function $f$ with the convex function $f(z)=\log \Phi_{i}\left(f_{z}\right)$ for $z=r, s, t$ in (1.8), where $r, s, t \in I$ such that $r<t<s$.

(ii) Since by $(i)$ the function $s \mapsto \Phi_{i}\left(f_{s}\right)$ is log-convex on $I$, that is, the function $s \mapsto \log \Phi_{i}\left(f_{s}\right)$ is convex on $I$. Applying Proposition 1.9 to $f(z)=\log \Phi_{i}\left(f_{z}\right)(i=1, \ldots, 6)$, we get

$$
\frac{\log \Phi_{i}\left(f_{s}\right)-\log \Phi_{i}\left(f_{q}\right)}{s-q} \leq \frac{\log \Phi_{i}\left(f_{u}\right)-\log \Phi_{i}\left(f_{v}\right)}{u-v}
$$

for $s \leq u, q \leq v, s \neq q, u \neq v$; and therefore, we conclude that

$$
\mu_{s, q}\left(\Phi_{i}, \Omega\right) \leq \mu_{u, v}\left(\Phi_{i}, \Omega\right), \quad i=1, \ldots, 6 .
$$

If $s=q$, we consider the limit when $q \rightarrow s$ in (3.4) and conclude that

$$
\mu_{s, s}\left(\Phi_{i}, \Omega\right) \leq \mu_{u, v}\left(\Phi_{i}, \Omega\right), \quad i=1, \ldots, 6 .
$$

The case $u=v$ can be treated similarly.

REMARK 3.14. Note that the results from Theorem 3.11, Corollary 3.12 and Corollary 3.13 still hold when two of the points $y_{0}, y_{1}, y_{2}, y_{3} \in[a, b]$ coincide, say $y_{1}=y_{0}$, for a family of differentiable functions $f_{s}$ such that the function $s \mapsto\left[y_{0}, y_{0}, y_{2}, y_{3} ; f_{s}\right]$ is $n$-exponentially convex in the Jensen sense (exponentially convex in the Jensen sense, log-convex in the Jensen sense on $\mathrm{I}$ ), when three of the points $y_{0}, y_{1}, y_{2}, y_{3} \in[a, b]$ coincide, say $y_{2}=y_{1}=y_{0}$, for a family of differentiable functions $f_{s}$ such that the function $s \mapsto\left[y_{0}, y_{0}, y_{0}, y_{3} ; f_{s}\right]$ is $n$-exponentially convex in the Jensen sense, when three of the points $y_{0}, y_{1}, y_{2}, y_{3} \in[a, b]$ coincide again, say $y_{2}=y_{1}=y_{0}$, for a family of twice differentiable functions $f_{s}$ such that the function $s \mapsto\left[y_{0}, y_{0}, y_{0}, y_{3} ; f_{s}\right]$ is $n$-exponentially convex in the Jensen sense and furthermore, they still hold when all four points coincide for a family of thrice differentiable functions with the same property. The proofs can be obtained by recalling Proposition 3.9 and by using suitable characterizations of convexity.

\section{EXAMPLES}

In this section, we present several families of functions which fulfil the conditions of Theorem 3.11, Corollary 3.12 and Corollary 3.13 and Remark 3.14. This enables us to construct large families of functions which are exponentially convex. 
EXAMPLE 4.1. Consider the family of functions

$$
\Omega_{1}=\left\{g_{s}: \mathbb{R} \rightarrow \mathbb{R}: s \in \mathbb{R}\right\}
$$

defined by

$$
g_{s}(x)= \begin{cases}\frac{1}{s^{3}} e^{s x}, & s \neq 0, \\ \frac{1}{6} x^{3}, & s=0 .\end{cases}
$$

We have $\frac{d^{3}}{d x^{3}} g_{s}(x)=e^{s x} \geq 0$, which shows that $g_{s}$ is 3 -convex on $\mathbb{R}$ for every $s \in \mathbb{R}$ and $s \mapsto \frac{d^{3}}{d x^{3}} g_{s}(x)$ is exponentially convex by definition (see [6]). It is easy to see that $s \mapsto\left[y_{0}, y_{1}, y_{2}, y_{3} ; g_{s}\right]$ is exponentially convex and so exponentially convex in the Jensen sense. Now by using Corollary 3.12, we have $s \mapsto \Phi_{i}\left(g_{s}\right)(i=1, \ldots, 6)$ are exponentially convex in the Jensen sense. Since these mappings are continuous (although the mapping $s \mapsto g_{s}$ is not continuous for $s=0)$, so $s \mapsto \Phi_{i}\left(g_{s}\right)(i=1, \ldots, 6)$ are exponentially convex.

For this family of functions, $\mu_{s, q}\left(\Phi_{i}, \Omega\right)(i=1, \ldots, 6)$ from (3.3) become

$$
\mu_{s, q}\left(\Phi_{i}, \Omega_{1}\right)= \begin{cases}\left(\frac{\Phi_{i}\left(g_{s}\right)}{\Phi_{i}\left(g_{q}\right)}\right)^{\frac{1}{s-q}}, & s \neq q, \\ \exp \left(\frac{\Phi_{i}\left(i d \cdot g_{s}\right)}{\Phi_{i}\left(g_{s}\right)}-\frac{3}{s}\right), & s=q \neq 0, \\ \exp \left(\frac{\Phi_{i}\left(i d \cdot g_{0}\right)}{4 \Phi_{i}\left(g_{0}\right)}\right), & s=q=0 .\end{cases}
$$

By using Theorem 2.8, it can be seen that

$$
M_{s, q}\left(\Phi_{i}, \Omega_{1}\right)=\log \mu_{s, q}\left(\Phi_{i}, \Omega_{1}\right), \quad i=1, \ldots, 6,
$$

satisfies $\min \{a, b\} \leq M_{s, q}\left(\Phi_{i}, \Omega_{1}\right) \leq \max \{a, b\}$, showing that $M_{s, q}\left(\Phi_{i}, \Omega_{1}\right)$ $(i=1, \ldots, 6)$ are means.

Example 4.2. Consider the family of functions

$$
\Omega_{2}=\left\{f_{s}:(0, \infty) \rightarrow \mathbb{R}: s \in \mathbb{R}\right\}
$$

defined by

$$
f_{s}(x)=\left\{\begin{aligned}
\frac{x^{s}}{s(s-1)(s-2)}, & s \neq 0,1,2, \\
\frac{1}{2} \ln x, & s=0, \\
-x \ln x, & s=1, \\
\frac{1}{2} x^{2} \ln x, & s=2 .
\end{aligned}\right.
$$

Here, $\frac{d^{3}}{d x^{3}} f_{s}(x)=x^{s-3}=e^{(s-3) \ln x}>0$, which shows that $f_{s}$ is 3 -convex for $x>0$ and $s \mapsto \frac{d^{3}}{d x^{3}} f_{s}(x)$ is exponentially convex by definition (see [6]). It is easy to prove that the function $s \mapsto\left[y_{0}, y_{1}, y_{2}, y_{3} ; f_{s}\right]$ is exponentially convex. Arguing as in Example 4.1, we have $s \mapsto \Phi_{i}\left(f_{s}\right)(i=1, \ldots, 6)$ are exponentially convex.

From (3.1), we have

$$
\Phi_{i}\left(f_{t}\right) \leq\left[\Phi_{i}\left(f_{r}\right)\right]^{\frac{s-t}{s-r}}\left[\Phi_{i}\left(f_{s}\right)\right]^{\frac{t-r}{s-r}}, \quad i=1, \ldots, 6,
$$


where $r, s, t \in I$ such that $r<t<s$. If $r<s<t$ or $t<r<s$, then opposite inequalities hold in (4.1).

If $r, s, t \in \mathbb{R} \backslash\{0,1,2\}$ such that $r<t<s$, then for $i=1,4$, (4.1) takes the form

$$
D_{s} \geq D_{r}^{\frac{t-s}{t-r}} D_{t}^{\frac{s-r}{t-r}}
$$

where $D_{s}$ denotes

$$
\begin{aligned}
D_{s}= & \frac{1}{s(s-1)(s-2)}\left(\left(\sum_{k=1}^{n} p_{k} a_{k}\right)^{s}-\left(p_{1} a_{1}\right)^{s}-\sum_{k=2}^{n} a_{k}^{s}\left(P_{k}^{s}-P_{k-1}^{s}\right)\right. \\
& \left.-s \sum_{k=2}^{n} a_{k}^{s-1} A_{k}\left(P_{k}^{s-1}-P_{k-1}^{s-1}\right)\right), \quad s \neq 0,1,2,
\end{aligned}
$$

where $P_{k-1}, P_{k}, P_{k-1} a_{k}, P_{k} a_{k}, \sum_{i=1}^{k-1} p_{i} a_{i}$ and $\sum_{i=1}^{k} p_{i} a_{i} \in[a, b]$ such that $a_{k}>0, p_{k}>0(k=1, \ldots, n)$ with $P_{k-1}=\sum_{i=1}^{k-1} p_{i} a_{i}$ and $A_{k}:=$ $\sum_{i=1}^{k-1} p_{i} a_{i}-P_{k-1} a_{k}$ for all $k=2, \ldots, n$. If we make the substitutions $p_{k} \rightarrow 1$ $(k=1, \ldots, n)$ in $(4.2)$, then the inequality obtained for $s>2$ is the refinement of the first inequality of (1.5).

For this family of functions by taking $\Omega=\Omega_{2}$ in (3.3), $\Xi_{s, q}^{i}:=\mu_{s, q}\left(\Phi_{i}, \Omega_{2}\right)$ $(i=1, \ldots, 6)$ for $x>0, x \in[a, b]$ are of the form

$$
\mu_{s, q}\left(\Phi_{i}, \Omega_{2}\right)= \begin{cases}\left(\frac{\Phi_{i}\left(f_{s}\right)}{\Phi_{i}\left(f_{q}\right)}\right)^{\frac{1}{s-q}}, & s \neq q, \\ \exp \left(\frac{2 \Phi_{i}\left(f_{s} f_{0}\right)}{\Phi_{i}\left(f_{s}\right)}-\frac{3 s^{2}-6 s+2}{s(s-1)(s-2)}\right), & s=q \neq 0,1,2, \\ \exp \left(\frac{\Phi_{i}\left(f_{0}^{2}\right)}{\Phi_{i}\left(f_{0}\right)}+\frac{3}{2}\right), & s=q=0, \\ \exp \left(\frac{\Phi_{i}\left(f_{0} f_{1}\right)}{\Phi_{i}\left(f_{1}\right)}\right), & s=q=1, \\ \exp \left(\frac{\Phi_{i}\left(f_{0} f_{2}\right)}{\Phi_{i}\left(f_{2}\right)}-\frac{3}{2}\right), & s=q=2 .\end{cases}
$$

In particular, for $i=1$ we have

$$
\Xi_{s, q}^{1}=\left(\frac{B_{s}}{B_{q}}\right)^{\frac{1}{s-q}}, \quad s \neq q
$$

where

$$
\begin{aligned}
B_{s}= & \frac{1}{s(s-1)(s-2)} \sum_{k=2}^{n}\left(\left(\sum_{i=1}^{k} p_{i} a_{i}\right)^{s}-\left(\sum_{i=1}^{k-1} p_{i} a_{i}\right)^{s}+a_{k}^{s}\left(P_{k-1}^{s}-P_{k}^{s}\right)\right. \\
& \left.+s a_{k}^{s-1} A_{k}\left(P_{k-1}^{s-1}-P_{k}^{s-1}\right)\right), \quad s \neq 0,1,2, \\
B_{0}= & \frac{1}{2} \sum_{k=2}^{n} a_{k}^{s}\left(\ln \left(\frac{P_{k-1} \sum_{i=1}^{k} p_{i} a_{i}}{P_{k} \sum_{i=1}^{k-1} p_{i} a_{i}}\right)+\frac{p_{k} A_{k}}{a_{k} P_{k} P_{k-1}}\right),
\end{aligned}
$$




$$
\begin{aligned}
B_{1}= & \sum_{k=2}^{n} a_{k}^{s}\left(\frac{\sum_{i=1}^{k-1} p_{i} a_{i}}{a_{k}} \ln \left(\frac{\sum_{i=1}^{k-1} p_{i} a_{i}}{a_{k}}\right)-\frac{\sum_{i=1}^{k} p_{i} a_{i}}{a_{k}} \ln \left(\frac{\sum_{i=1}^{k} p_{i} a_{i}}{a_{k}}\right)\right. \\
& \left.-P_{k-1} \ln P_{k-1}+P_{k} \ln P_{k}+\frac{A_{k}}{a_{k}} \ln \frac{P_{k}}{P_{k-1}}\right), \\
B_{2}= & \frac{1}{2} \sum_{k=2}^{n} a_{k}^{s}\left(\left(\frac{\sum_{i=1}^{k} p_{i} a_{i}}{a_{k}}\right)^{2} \ln \left(\frac{\sum_{i=1}^{k} p_{i} a_{i}}{a_{k}}\right)\right. \\
& -\left(\frac{\sum_{i=1}^{k-1} p_{i} a_{i}}{a_{k}}\right)^{2} \ln \left(\frac{\sum_{i=1}^{k-1} p_{i} a_{i}}{a_{k}}\right)+P_{k-1}^{2} \ln P_{k-1}-P_{k}^{2} \ln P_{k} \\
& \left.-\frac{A_{k}\left(p_{k}+2\left(P_{k} \ln P_{k}-P_{k-1} \ln P_{k-1}\right)\right)}{a_{k}}\right) .
\end{aligned}
$$

Denoting, further,

$$
\begin{aligned}
C_{s}= & \sum_{k=2}^{n} \frac{a_{k}^{s}}{s(s-1)(s-2)}\left(\left(\frac{\sum_{i=1}^{k} p_{i} a_{i}}{a_{k}}\right)^{s} \ln \left(\frac{\sum_{i=1}^{k} p_{i} a_{i}}{a_{k}}\right)\right. \\
& -\left(\frac{\sum_{i=1}^{k-1} p_{i} a_{i}}{a_{k}}\right)^{s} \ln \left(\frac{\sum_{i=1}^{k-1} p_{i} a_{i}}{a_{k}}\right)+P_{k-1}^{s} \ln P_{k-1}-P_{k}^{s} \ln P_{k} \\
& \left.+\frac{A_{k}\left(P_{k-1}^{s-1}\left(1+s \ln P_{k-1}\right)-P_{k}^{s-1}\left(1+s \ln P_{k}\right)\right)}{a_{k}}\right), \\
& s \neq 0,1,2, \\
C_{0}= & \frac{1}{4} \sum_{k=2}^{n} a_{k}^{s}\left(\ln ^{2}\left(\frac{\sum_{i=1}^{k} p_{i} a_{i}}{a_{k}}\right)-\ln ^{2}\left(\frac{\sum_{i=1}^{k-1} p_{i} a_{i}}{a_{k}}\right)+\ln ^{2}\left(P_{k-1}\right)\right. \\
& \left.-\ln ^{2}\left(P_{k}\right)-2 \frac{A_{k}}{a_{k}}\left(\frac{\ln P_{k}}{P_{k}}-\frac{\ln P_{k-1}}{P_{k-1}}\right)\right), \\
C_{1}= & \frac{1}{2} \sum_{k=2}^{n} a_{k}^{s}\left(\left(\frac{\sum_{i=1}^{k-1} p_{i} a_{i}}{a_{k}}\right) \ln ^{2}\left(\frac{\sum_{i=1}^{k-1} p_{i} a_{i}}{a_{k}}\right)\right. \\
& -\left(\frac{\sum_{i=1}^{k} p_{i} a_{i}}{a_{k}}\right) \ln ^{2}\left(\frac{\sum_{i=1}^{k} p_{i} a_{i}}{a_{k}}\right)-P_{k-1} \ln ^{2}\left(P_{k-1}\right)+P_{k} \ln ^{2}\left(P_{k}\right) \\
& \left.+\frac{A_{k}\left(\left(2+\ln P_{k}\right) \ln P_{k}-\left(2+\ln P_{k-1}\right) \ln _{k-1}\right)}{a_{k}}\right),
\end{aligned}
$$




$$
\begin{aligned}
C_{2}= & \frac{1}{4} \sum_{k=2}^{n} a_{k}^{s}\left(\left(\frac{\sum_{i=1}^{k} p_{i} a_{i}}{a_{k}}\right)^{2} \ln ^{2}\left(\frac{\sum_{i=1}^{k} p_{i} a_{i}}{a_{k}}\right)\right. \\
& -\left(\frac{\sum_{i=1}^{k-1} p_{i} a_{i}}{a_{k}}\right)^{2} \ln ^{2}\left(\frac{\sum_{i=1}^{k-1} p_{i} a_{i}}{a_{k}}\right)+P_{k-1}^{2} \ln ^{2} P_{k-1}-P_{k}^{2} \ln ^{2} P_{k} \\
& \left.+\frac{2 A_{k}\left(P_{k-1}\left(1+\ln P_{k-1}\right) \ln P_{k-1}-P_{k}\left(1+\ln P_{k}\right) \ln P_{k}\right)}{a_{k}}\right)
\end{aligned}
$$

we can express $\Xi_{s, s}^{1}$ as

$$
\begin{aligned}
& \Xi_{s, s}^{1}=\exp \left(\frac{C_{s}}{B_{s}}-\frac{3 s^{2}-6 s+2}{s(s-1)(s-2)}\right), \quad s \neq 0,1,2, \\
& \Xi_{0,0}^{1}=\exp \left(\frac{C_{0}}{B_{0}}+\frac{3}{2}\right), \\
& \Xi_{1,1}^{1}=\exp \left(\frac{C_{1}}{B_{1}}\right) \\
& \Xi_{2,2}^{1}=\exp \left(\frac{C_{2}}{B_{2}}-\frac{3}{2}\right) .
\end{aligned}
$$

If $\Phi_{i}(i=1, \ldots, 6)$ are positive, then Theorem 2.8 applied for $g=f_{s} \in \Omega_{2}$ and $h=f_{q} \in \Omega_{2}$ yields that there exists $\xi_{i} \in[a, b]$ such that

$$
\xi_{i}^{s-q}=\frac{\Phi_{i}\left(f_{s}\right)}{\Phi_{i}\left(f_{q}\right)}, \quad i=1, \ldots, 6 .
$$

Since the functions $\xi_{i} \mapsto \xi_{i}^{s-q}(i=1, \ldots, 6)$ are invertible for $s \neq q$, we have

$$
\min \{a, b\} \leq\left(\frac{\Phi_{i}\left(f_{s}\right)}{\Phi_{i}\left(f_{q}\right)}\right)^{\frac{1}{s-q}} \leq \max \{a, b\}, \quad i=1, \ldots, 6
$$

which together with the fact that $\mu_{s, q}\left(\Phi_{i}, \Omega_{2}\right)(i=1, \ldots, 6)$ are continuous, symmetric and monotonous (by $(3.2))$ shows that $\mu_{s, q}\left(\Phi_{i}, \Omega_{2}\right)(i=1, \ldots, 6)$ are means.

EXAMPLE 4.3. Consider the family of functions $\Omega_{3}=\left\{h_{s}:(0, \infty) \rightarrow \mathbb{R}:\right.$ $s \in(0, \infty)\}$ defined by

$$
h_{s}(x)=\left\{\begin{array}{cl}
-\frac{s^{-x}}{\ln ^{3} s}, & s \neq 1 \\
\frac{x^{3}}{6}, & s=1 .
\end{array}\right.
$$

We have $\frac{d^{3}}{d x^{3}} h_{s}(x)=s^{-x}>0$, which shows that $h_{s}$ is 3-convex for all $s>0$. Since $s \mapsto \frac{d^{3}}{d x^{3}} h_{s}(x)=s^{-x}$ is the Laplace transform of a non-negative function (see $[6,11])$, it is exponentially convex. It is easy to see that the function $s \mapsto\left[y_{0}, y_{1}, y_{2}, y_{3} ; h_{s}\right]$ is also exponentially convex. Arguing as in Example 4.1 , we have $s \mapsto \Phi_{i}\left(h_{s}\right)(i=1, \ldots, 6)$ are exponentially convex. 
In this case, by taking $\Omega=\Omega_{3}$ in $(3.3), \mu_{s, q}\left(\Phi_{i}, \Omega\right)(i=1, \ldots, 6)$ for $x>0$, where $x \in[a, b]$, are of the form

$$
\mu_{s, q}\left(\Phi_{i}, \Omega_{3}\right)= \begin{cases}\left(\frac{\Phi_{i}\left(h_{s}\right)}{\Phi_{i}\left(h_{q}\right)}\right)^{\frac{1}{s-q}}, & s \neq q, \\ \exp \left(-\frac{\Phi_{i}\left(i d \cdot h_{s}\right)}{s \Phi_{i}\left(h_{s}\right)}-\frac{3}{s \ln s}\right), & s=q \neq 1, \\ \exp \left(-\frac{\Phi_{i}\left(i d \cdot h_{1}\right)}{4 \Phi_{i}\left(h_{1}\right)}\right), & s=q=1 .\end{cases}
$$

By using Theorem 2.8, it follows that

$$
M_{s, q}\left(\Phi_{i}, \Omega_{3}\right)=-L(s, q) \log \mu_{s, q}\left(\Phi_{i}, \Omega_{3}\right), \quad i=1, \ldots, 6,
$$

satisfy $\min \{a, b\} \leq M_{s, q}\left(\Phi_{i}, \Omega_{3}\right) \leq \max \{a, b\}$ and so $M_{s, q}\left(\Phi_{i}, \Omega_{3}\right)$ are means $(i=1, \ldots, 6)$, where $L(s, q)$ is the logarithmic mean defined by $L(s, q)=$ $\frac{s-q}{\log s-\log q}, s \neq q, L(s, s)=s$.

EXAmple 4.4. Consider the family of functions $\Omega_{4}=\left\{k_{s}:(0, \infty) \rightarrow\right.$ $(0, \infty): s \in(0, \infty)\}$ defined by

$$
k_{s}(x)=\frac{e^{-x \sqrt{s}}}{\sqrt{s^{3}}} .
$$

Here, $\frac{d^{3}}{d x^{3}} k_{s}(x)=e^{-x \sqrt{s}}>0$, which shows that $k_{s}$ is 3 -convex for all $s>0$. Since $s \mapsto \frac{d^{3}}{d x^{3}} k_{s}(x)=e^{-x \sqrt{s}}$ is the Laplace transform of a non-negative function (see $[6,11]$ ), it is exponentially convex. It is easy to see that the function $s \mapsto\left[y_{0}, y_{1}, y_{2}, y_{3} ; k_{s}\right]$ is also exponentially convex. Arguing as in Example 4.1, we have $s \mapsto \Phi_{i}\left(k_{s}\right)(i=1, \ldots, 6)$ are exponentially convex.

In this case, by taking $\Omega=\Omega_{4}$ in $(3.3), \mu_{s, q}\left(\Phi_{i}, \Omega\right)(i=1, \ldots, 6)$ for $x>0$, where $x \in[a, b]$ are of the form

$$
\mu_{s, q}\left(\Phi_{i}, \Omega_{4}\right)= \begin{cases}\left(\frac{\Phi_{i}\left(k_{s}\right)}{\Phi_{i}\left(k_{q}\right)}\right)^{\frac{1}{s-q}}, & s \neq q, \\ \exp \left(-\frac{\Phi_{i}\left(i d \cdot k_{s}\right)}{2 \sqrt{s} \Phi_{i}\left(k_{s}\right)}-\frac{3}{2 s}\right), & s=q .\end{cases}
$$

By using Theorem 2.8, it is easy to see that

$$
M_{s, q}\left(\Phi_{i}, \Omega_{4}\right)=-(\sqrt{s}+\sqrt{q}) \log \mu_{s, q}\left(\Phi_{i}, \Omega_{4}\right), \quad i=1, \ldots, 6,
$$

satisfies $\min \{a, b\} \leq M_{s, q}\left(\Phi_{i}, \Omega_{4}\right) \leq \max \{a, b\}$, showing that $M_{s, q}\left(\Phi_{i}, \Omega_{4}\right)$ $(i=1, \ldots, 6)$ are means.

Remark 4.5. From (3.2), it is clear that $\mu_{s, q}\left(\Phi_{i}, \Omega\right)(i=1, \ldots, 6)$ for $\Omega=\Omega_{1}, \Omega_{3}$ and $\Omega_{4}$ are monotonous functions in parameters $s$ and $q$.

ACKNOWLEDGEMENTs.

This research work was partially supported by Higher Education Commission, Pakistan. The second author's research was supported by the Croatian Ministry of Science, Education and Sports, under the Research Grant 1171170889-0888. 


\section{REFERENCES}

[1] M. Anwar and J. Pečarić, Means of the Cauchy type, LAP Lambert Academic Publishing, 2009.

[2] G. Bennett, Lower bounds for matrices, Linear Algebra Appl. 82 (1986), 81-98.

[3] G. H. Hardy, J. E. Littlewood and G. Pólya, Inequalities, Cambridge Univ. Press, Cambridge, 1952.

[4] E. Isaacson and H. B. Keller, Analysis of numerical methods, Dover publications Inc., New York, 1966.

[5] C. Jardas, J. Pečarić, R. Roki and N. Sarapa, On an inequality of Hardy-LittlewoodPólya and some applications to entropies, Glas. Mat. Ser. III 32(52) (1997), 201-206.

[6] J. Jakšetić and J. Pečarić, Exponential convexity method, J. Convex Anal. 20 (2013), 181-197.

[7] Ž. Pauše, Uvod u teoriju informacije, Šk. knjiga, Zagreb, 1989.

[8] J. Pečarić, F. Proschan and Y. L. Tong, Convex functions, partial orderings, and statistical applications, Academic Press Inc., 1992.

[9] J. Pečarić and L. E. Persson, On an inequality of Hardy-Littlewood-Pólya, Math. Gazette 79 (1995), 383-385.

[10] J. Pečarić and J. Perić, Improvements of the Giaccardi and the Petrović inequality and related Stolarsky type means, An. Univ. Craiova Ser. Mat. Inform. 39 (2012), 65-75.

[11] D. V. Widder, The Laplace transform, Princeton Univ. Press, Princeton, 1941.

S. Khalid

Abdus Salam School of Mathematical Sciences

GC University

68-B, New Muslim Town, Lahore 54600

Pakistan

E-mail: saadiakhalid176@gmail.com

J. Pečarić

Abdus Salam School of Mathematical Sciences

GC University

68-B, New Muslim Town, Lahore 54600

Pakistan

and

Faculty of Textile Technology

University of Zagreb

Prilaz baruna Filipovića 28a, 10000 Zagreb

Croatia

E-mail: pecaric@element.hr

M. Praljak

Abdus Salam School of Mathematical Sciences

GC University

68-B, New Muslim Town, Lahore 54600

Pakistan

and

Faculty of Food Technology and Biotechnology

University of Zagreb

Pierottijeva 6, 10000 Zagreb

Croatia

E-mail: mpraljak@pbf.hr

Received: 1.2.2013. 